Supporting Information for the manuscript

\title{
Mixed Uranyl Sulfate-Selenates: Evolution of Structural Topology and Complexity vs. Chemical Composition
}

by

Vladislav V. Gurzhiy,,$^{* \dagger}$ Olga S. Tyumentseva,${ }^{\dagger}$ Sergey V. Krivovichev, ${ }^{\dagger}$ Vladimir G. Krivovichev, ${ }^{\dagger}$ and Ivan G. Tananaev ${ }^{\ddagger}$

$\dagger$ Institute of Earth Sciences, St. Petersburg State University, 199034, University emb. 7/9, St. Petersburg, Russian Federation

Far Eastern Federal University, 690950, Suhanova st. 8, Vladivostok, Russian Federation 


\section{EDX spectra.}

\section{Compound 3}

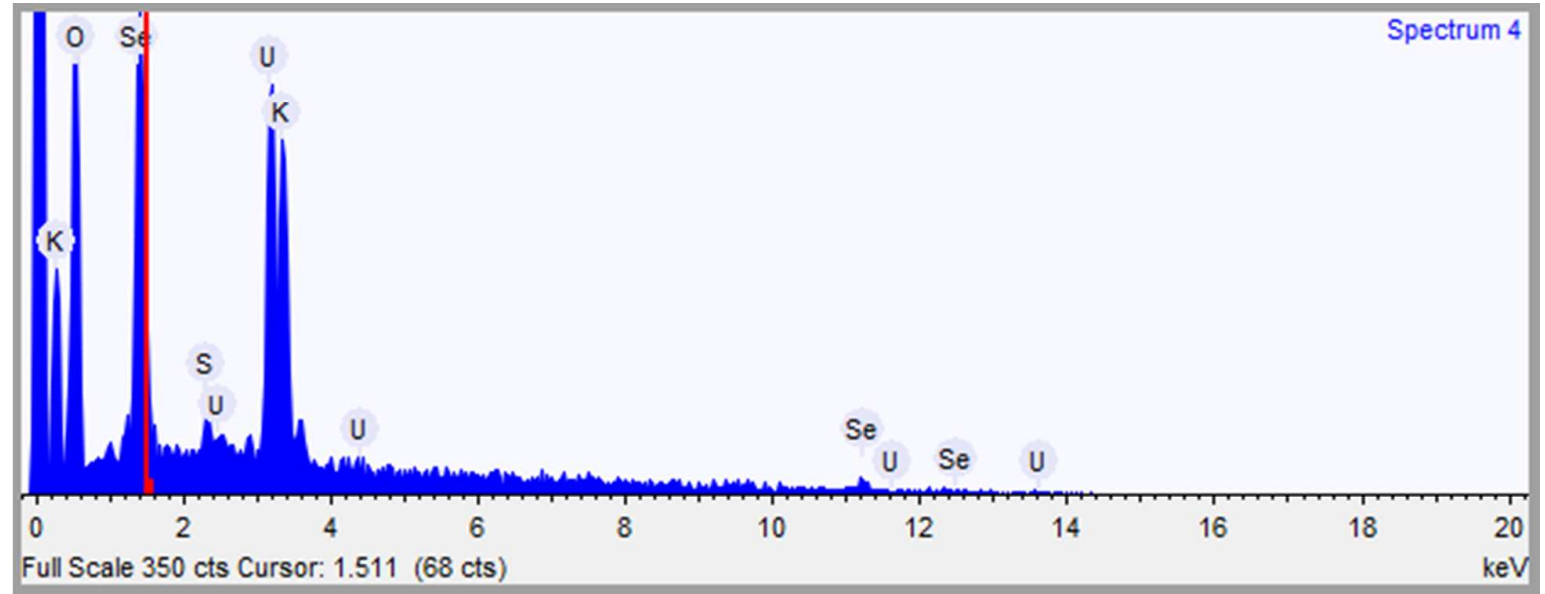

Analytical calculations: atomic ratio from structural data K 1.00, U 2.00, S 0.30 , Se 2.70; found by EDX: $K$ 0.88, U 2.12, S 0.32, Se 2.67.

\section{Compound 4}

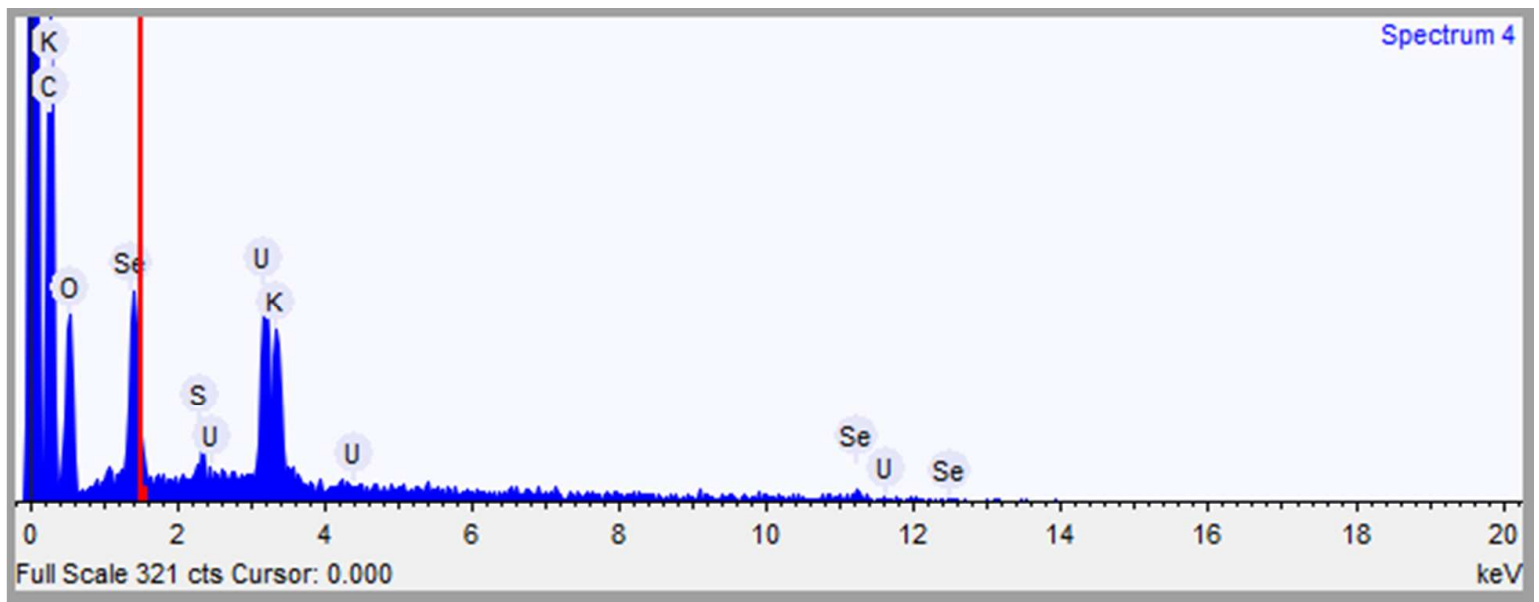

Analytical calculations: atomic ratio from structural data K 1.00, U 2.00, S 0.60, Se 2.40; found by EDX: K 0.96 , U 2.15, S 0.55, Se 2.34. 


\section{Compound 11}

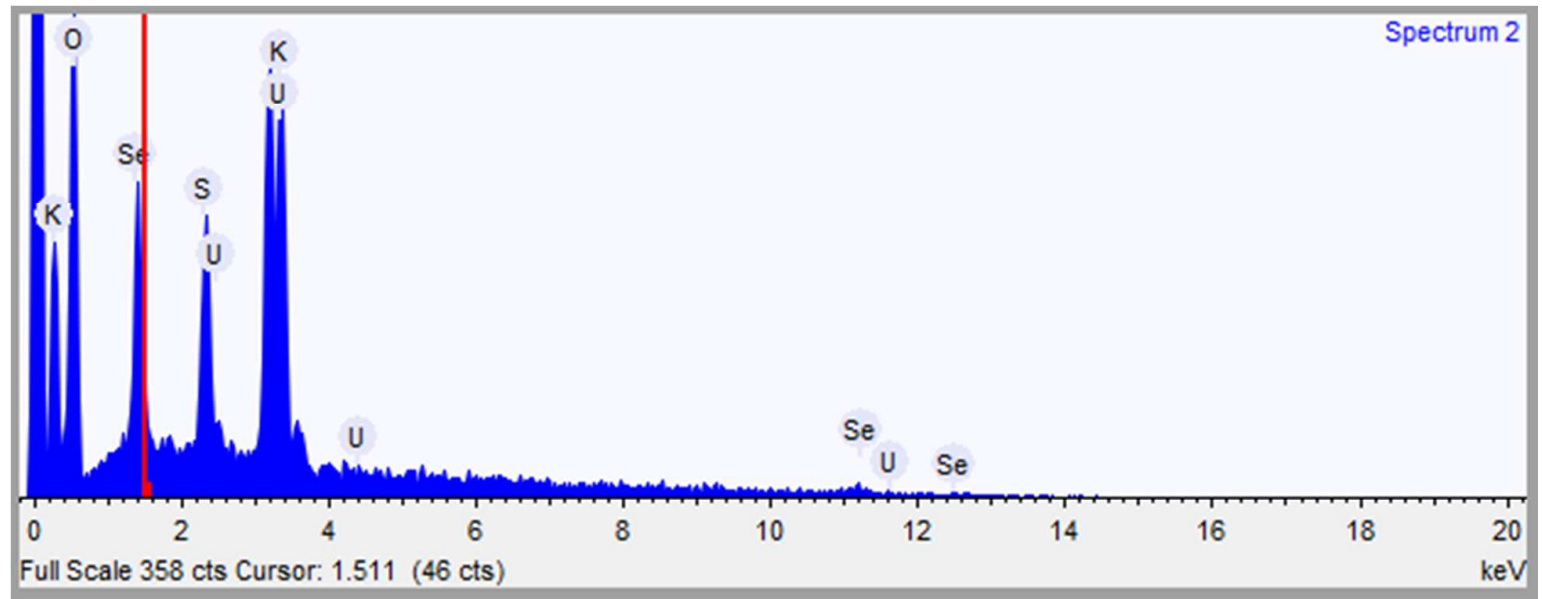

Analytical calculations: atomic ratio from structural data K 5.00, U 6.00, S 6.30, Se 4.70; found by EDX: $K$ 4.69, U 6.35, S 6.39, Se 4.57.

\section{Compound 13}

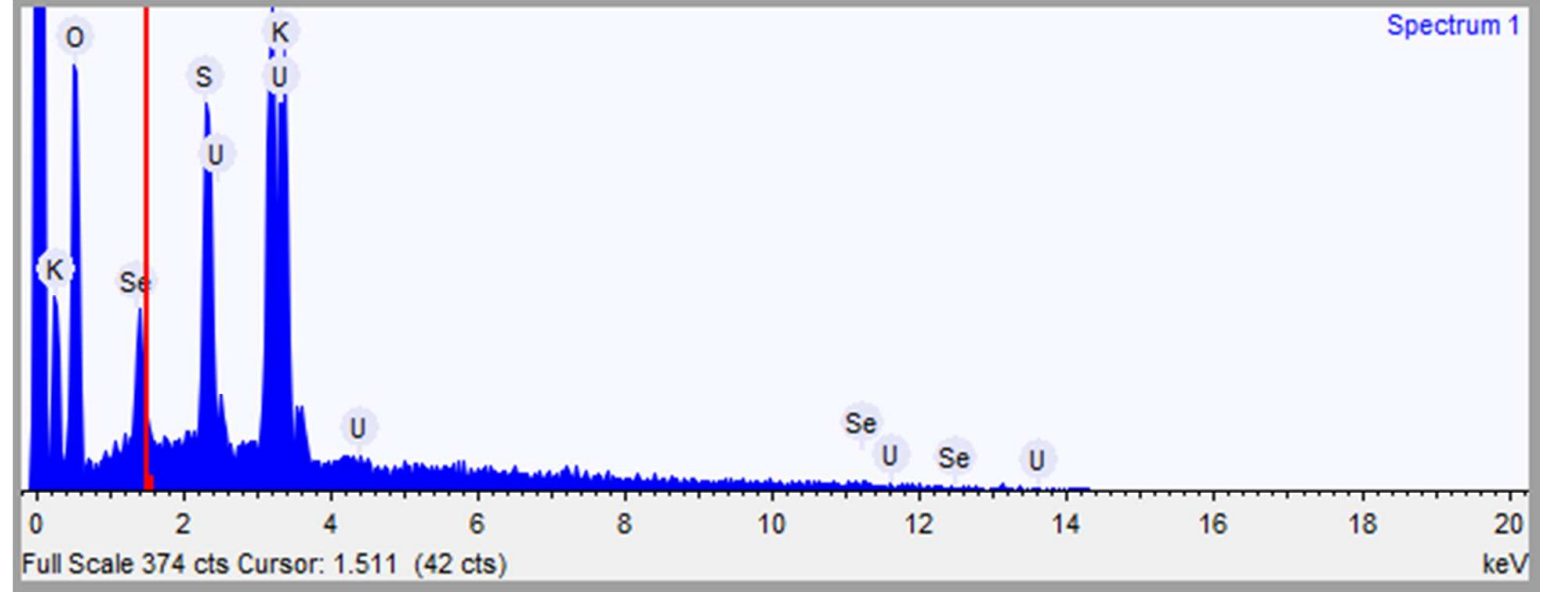

Analytical calculations: atomic ratio from structural data K 5.00, U 6.00, S 8.10, Se 2.90; found by EDX: K 5.18, U 6.16, S 8.35, Se 2.31. 


\section{Compound 14}

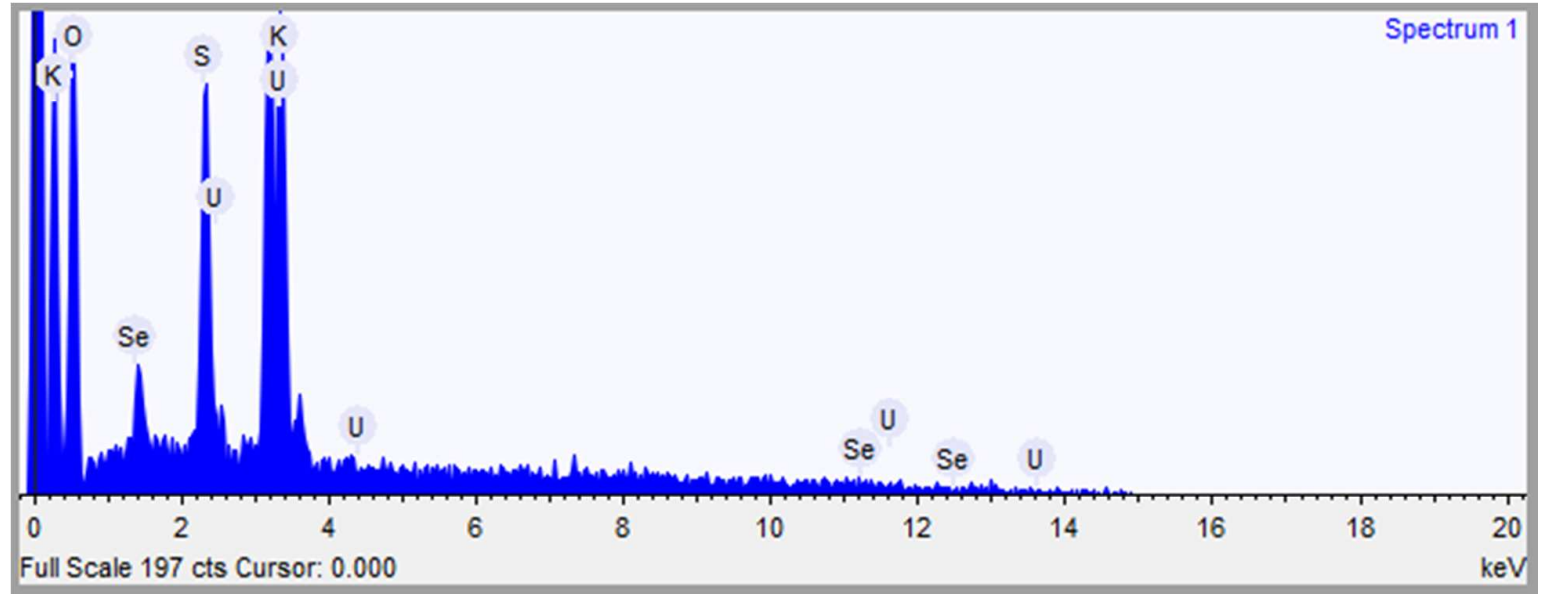

Analytical calculations: atomic ratio from structural data K 5.00, U 6.00, S 9.40, Se 1.60; found by EDX: K 5.03, U 6.04, S 9.43, Se 1.50 .

\section{Compound 15}

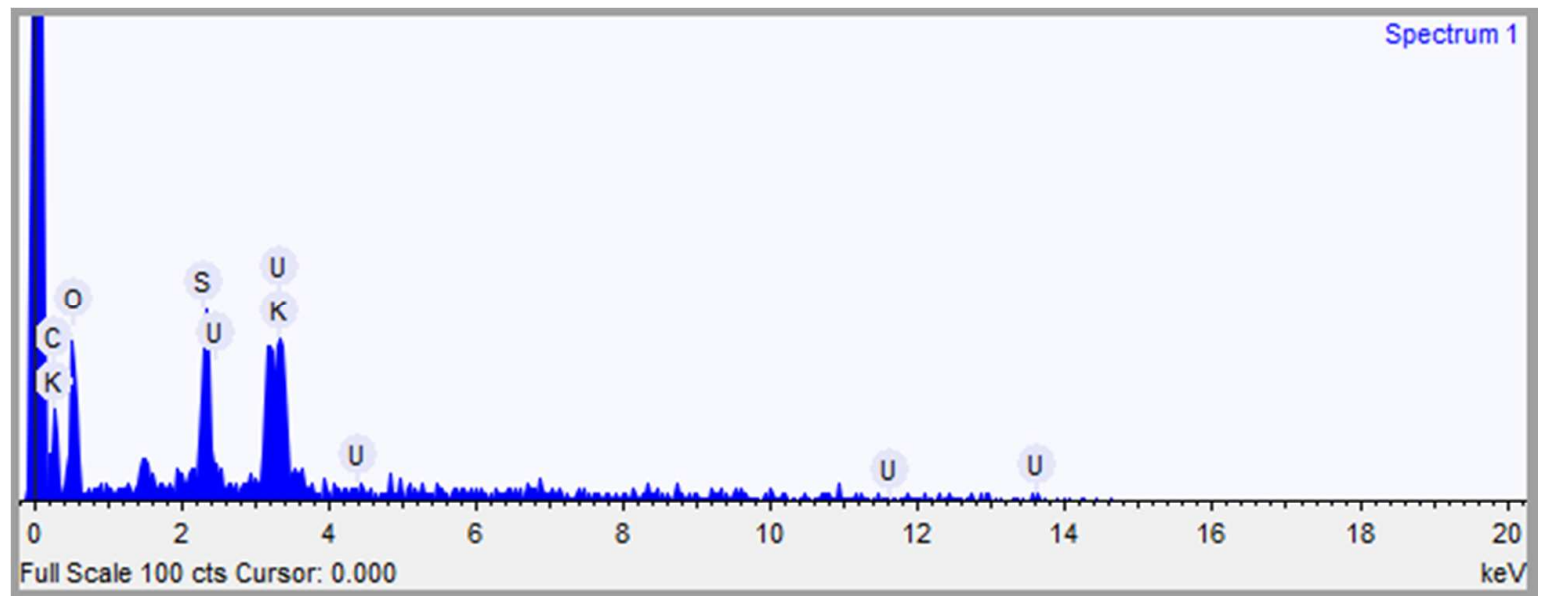

Analytical calculations: atomic ratio from structural data K 5.00, U 6.00, S 11.00; found by EDX: K 4.97, U 6.12, S 10.91. 